\title{
A consistent approximation scheme beyond RPA for bosons
}

\author{
D. Davesne, M. Oertel, H. Hansen
}

November 6, 2018

\begin{abstract}
In this paper, we develop a consistent extension of RPA for bosonic systems. In order to illustrate the method, we consider the case of the anharmonic oscillator. We compare our results with those obtained in mean-field and standard RPA approaches, with the exact ones and show that they are very close to the exact ones.
\end{abstract}

IPN Lyon, 43 Bd du 11 Novembre 1918, F-69622 Villeurbanne Cedex

LYCEN 2002-30

\section{Introduction}

In many domains of physics perturbation theory fails to describe physical phenomena correctly. For example, bound states or collective excitations cannot be described perturbatively. In particular in the low-energy regime of strong interactions non-perturbative effects play an important role. Although much progress has been made in understanding the hadronic world, it is still indispensable to develop non-perturbative, symmetry conserving methods.

In the last few years much effort has been made to apply non-perturbative techniques well established in many-body theory to quantum field theory (see, e.g., Refs. [1, 2, 3, [4]). In this article we will concentrate on approaches related to the random phase approximation (RPA). In its standard form the RPA is known to respect symmetries. For example, in Ref. [2] it has been demonstrated within a linear $\sigma$-model that the RPA fluctuations generate 
the Goldstone bosons related to the spontaneously broken global (chiral) symmetry. At mean-field level, on the contrary, these soft modes do not emerge. Thus the RPA seems to be a method very well suited to treat nonperturbative systems with spontaneously broken symmetries. Nevertheless it has some shortcomings: It is not of variational character and therefore does not necessarily fulfill the Rayleigh-Ritz criterion. In addition, it is not self-consistent, and therefore the intermediate states (corresponding to the mean-field quasiparticles) do not coincide with the final RPA particles. In particular, even if the RPA states show up as massless Goldstone modes, the intermediate states are massive. This has been pointed out by Aouissat et al. [3, [0] in the context of pionic excitations in an $O(N)$-model.

It is obvious that this self-consistency problem can be circumvented in $1 / N$-expansions [3, 5, 6]. On the other hand, a fully self-consistent approximation such as Self-Consistent RPA (SCRPA) [7] is desirable. This method has already been applied successfully to fermionic systems, e.g., to the Hubbard model [8] and the seniority model [9]. For a bosonic field theory a first attempt in this direction has been made in Ref. [4]. There some of the correlations are included, which are not present in the mean-field ground state used in standard RPA. This procedure, however, causes serious problems: In particular it does not preserve covariance, an essential ingredient of a relativistic quantum field theory.

In order to establish a consistent method and to clearly state the problems related to the treatment of a bosonic system in an extended RPA formalism we will reduce the dimension of the system. Instead of a $\lambda \Phi^{4}$-theory as in Ref. 团 we will study a one-dimensional anharmonic oscillator. Of course this is a purely quantum mechanical, non-relativistic system and we can certainly not tackle the problem of covariance. Nevertheless, we can isolate some of the inherent contradictions related to bosonic systems. It has to be examined within a future work whether the method presented here is able to preserve covariance.

Our toy model, the anharmonic oscillator has the advantage, that exact results are known and that we can directly compare the results obtained in different approximation schemes with those obtained by solving the Schrödinger equation. It has already been used as a test ground, e.g., for coupled cluster techniques [10] or first attempts within SCRPA [11].

The article is organized as follows: We begin with presenting the RPA equations in Sec. 2. In Sec. 3, we review the Hartree-Fock (mean-field) approximation together with the standard RPA. The renormalized RPA ( $\mathrm{r}-$ 


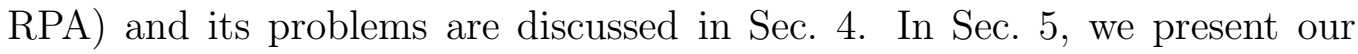
extended r-RPA approach. Sec. 6 is devoted to a discussion of the numerical results, in particular for the ground state energy, obtained within the different approximation schemes. Finally, in Sec. 7, we will draw our conclusions.

\section{The RPA equations}

First of all, we have to state our starting point, the RPA equations. There are mainly two different ways to derive the RPA equations: the equation of motion method due to Rowe [12 and the Dyson-Schwinger-approach [13]. To derive the former, we suppose that we can generate an excited state $|\nu\rangle$ by the action of an operator $Q_{\nu}^{\dagger}$ on the ground state $|\mathrm{gs}\rangle:|\nu\rangle=Q_{\nu}^{\dagger}|\mathrm{gs}\rangle$, $\mid$ gs $\rangle$ being the vacuum of $Q_{\nu}$. Obviously $Q_{\nu}^{\dagger}$ is a highly non-trivial manybody operator. Depending on the nature of $|\nu\rangle$ (excited state with the same number of particles or excited state with a different number of particles), $Q_{\nu}^{\dagger}$ can be seen as a superposition of (non-hermitian) one, two or more body operators. In general, $Q_{\nu}^{\dagger}$ is expressed in the following form:

$$
Q_{\nu}^{\dagger}=\sum_{\alpha} X_{\alpha}^{\nu} A_{\alpha}^{\dagger}
$$

where $A_{\alpha}^{\dagger}$ contains all the chosen excitation operators expressed in terms of $a_{i}$ and $a_{i}^{\dagger}$ (annihilation and creation operators) and $\alpha$ represents all quantum numbers. Moreover, minimization of the energy $E_{\nu}=\langle\nu|\mathrm{H}| \nu\rangle$ with respect to a variation $\delta Q_{\nu}$ leads to a system of equations whose solution gives the amplitudes $X_{\alpha}^{\nu}$ and the excitation energies:

$$
\left\langle\mathrm{gs}\left|\left[\delta Q_{\nu},\left[\mathrm{H}, Q_{\nu}^{\dagger}\right]\right]\right| \mathrm{gs}\right\rangle=\left(E_{\nu}-E_{0}\right)\left\langle\mathrm{gs}\left|\left[\delta Q_{\nu}, Q_{\nu}^{\dagger}\right]\right| \mathrm{gs}\right\rangle,
$$

where $E_{0}$ represents the energy of the ground state $|\mathrm{gs}\rangle$.

In the Dyson-Schwinger approach, which will be used in this paper, one starts from the definition of a time-ordered Green's function at zero temperature in equilibrium (the generalization to finite temperature is direct):

$$
G_{\alpha \beta}\left(t, t^{\prime}\right)=-i\left\langle\mathrm{gs}\left|T\left(A_{\alpha}(t) A_{\beta}^{\dagger}\left(t^{\prime}\right)\right)\right| \mathrm{gs}\right\rangle,
$$

where $T$ is the time ordering operator and $A$ and $A^{\dagger}$ depend on time via $A(t)=e^{i H t} A(0) e^{-i H t}$, where $\mathrm{H}$ is the exact Hamiltonian. In equilibrium the 
above two-time Green's function' is a function of the time difference $t-t^{\prime}$ so that the Fourier transform $G(E)$ depends only on one frequency. Our aim is to derive a Dyson equation for $G(E)$. If we define

$$
G_{\alpha \beta}\left(t, t^{\prime}\right)=\int \frac{d E}{2 \pi} e^{-i E\left(t-t^{\prime}\right)} G_{\alpha \beta}(E)
$$

the result is

$$
E G_{\alpha \beta}(E)=\mathcal{N}_{\alpha \beta}+\sum_{\beta^{\prime}, \gamma} \mathcal{D} \mathcal{C}_{\alpha \beta^{\prime}} \mathcal{N}_{\beta^{\prime} \gamma}^{-1} G_{\gamma \alpha}(E)
$$

with

$$
\begin{aligned}
\mathcal{N}_{\alpha \beta} & =\left\langle\mathrm{gs}\left|\left[A_{\alpha}, A_{\beta}^{\dagger}\right]\right| \mathrm{gs}\right\rangle \\
\mathcal{D C}_{\alpha \beta} & =\left\langle\mathrm{gs}\left|\left[\left[A_{\alpha}, \mathrm{H}\right], A_{\beta}^{\dagger}\right]\right| \mathrm{gs}\right\rangle
\end{aligned}
$$

It has to be noted that the operators entering the above Eq. (6) have to be taken at equal times since the truly dynamical contribution has been omitted (see, e.g., the derivation in Refs. 8, 14).

One can show that the two methods mentioned above are strictly equivalent provided that [14]

$$
\left\langle\mathrm{gs}\left|\left[\mathrm{H},\left[A_{\alpha}, A_{\beta}^{\dagger}\right]\right]\right| \mathrm{gs}\right\rangle=0 .
$$

Of course, if $|g s\rangle$ is the exact ground state, i.e., an eigenstates of the Hamiltonian, this relation is automatically satisfied. Within an approximation this needs, however, not to be the case. Nevertheless, in most practical situations, including our treatment of the anharmonic oscillator, it can be shown that both methods are indeed equivalent.

Obviously it is not possible to include the complete Hilbert space of excitation operators in $A$ and it is necessary to restrict it. In principle the approximation can straightforwardly be refined by enlarging the space of excitation operators contained in $A$. But, in practice this often turns out to be a very difficult task because, on one hand, the dimension of the RPA matrix to be diagonalized increases and, on the other hand, there exist consistency problems for the theory itself (see the next section).

\footnotetext{
${ }^{1}$ Note that we deal with a two-time Green's function. That means that, if, e.g., $A=$ $a^{\dagger} a^{\dagger}$, both particles are created at the same time.
} 
Another crucial point in solving the above RPA equations, Eq. (5), is the way in which the expectation values are determined since the exact ground state is not known. It is however clear that it strongly influences the quality of the approximation. In standard RPA the exact ground state is usually replaced by a mean-field (Hartree-Fock) one. But we will show in the next section, using as an example the anharmonic oscillator, that it is possible to take into account an important part of the correlations present in the exact ground state in a fully consistent way.

\section{The model in standard RPA}

We start from the Hamiltonian of an anharmonic oscillator with quartic coupling:

$$
\mathrm{H}=\frac{p^{2}}{2}+\mu^{2} \frac{X^{2}}{2}+g X^{4} .
$$

with constants $\mu^{2}<0$ and $g>0$. In the following sections, $\tilde{\mu} \equiv \sqrt{\left|\mu^{2}\right|}$. This Hamiltonian can be rewritten as

$$
\mathrm{H}=\frac{p^{2}}{2}+\frac{X^{2}}{2}+\left(\mu^{2}-1\right) \frac{X^{2}}{2}+g X^{4}
$$

and can be seen as a harmonic oscillator disturbed by a potential $V(X)=$ $\left(\mu^{2}-1\right) \frac{X^{2}}{2}+g X^{4}$. We introduce the usual destruction and creation operators $a$ and $a^{\dagger}$ defined by $a=(\tilde{\mu} X+i p) / \sqrt{2 \tilde{\mu}}$. The basis for standard RPA is the mean-field approximation which is usually formulated with the help of a Bogoliubov transformation. Thereby the starting point is the following trial wave function (see, e.g., Ref. [10]):

$$
|\mathrm{HF}\rangle=e^{\left(u a^{\dagger}+\frac{1}{2} t a^{\dagger 2}\right)}|V\rangle,
$$

with variational parameters $u$ and $t .|V\rangle$ is the vacuum of the initial destruction operator $a: a|V\rangle=0$. The destruction operator of the Hartree-Fock vacuum $b|\mathrm{HF}\rangle=0$ can be expressed with the help of $u$ and $t$ :

$$
b=\left(1-t^{2}\right)^{-1 / 2}\left(a-t a^{\dagger}-u\right) .
$$

For later convenience we define $\omega \equiv \tilde{\mu}(1-t) /(1+t)$ and $s \equiv \sqrt{2 / \tilde{\mu}} u /(t-1)$. In terms of the new creation and destruction operators we then obtain

$$
x \equiv X+s=\frac{1}{\sqrt{2 \omega}}\left(b+b^{\dagger}\right)
$$




$$
p=-i \sqrt{\frac{\omega}{2}}\left(b-b^{\dagger}\right)
$$

which makes the role of the variational parameters obvious: $s$ simply describes a translation in space and $\omega$ a modified frequency of the oscillator. The Hamiltonian can be cast into the following form:

$$
\begin{array}{r}
\mathrm{H}=-\frac{\omega}{4}\left(b-b^{\dagger}\right)^{2}+\frac{\mu^{2}+12 g s^{2}}{4 \omega}\left(b+b^{\dagger}\right)^{2} \\
-\frac{s \mu^{2}+4 g s^{3}}{\sqrt{2 \omega}}\left(b+b^{\dagger}\right)-\frac{4 s g}{\sqrt{8 \omega^{3}}}\left(b+b^{\dagger}\right)^{3}+\frac{g}{4 \omega^{2}}\left(b+b^{\dagger}\right)^{4} \\
+g s^{4}+\frac{\mu^{2} s^{2}}{2} .
\end{array}
$$

The aim of the Bogoliubov transformation is to select the best basis in the sense that the parameters $\omega$ and $s$ minimize the mean-field ground state energy, $E_{0}^{H F}=\langle\mathrm{HF}|\mathrm{H}| \mathrm{HF}\rangle$. These two parameters are thus determined by: $\frac{\partial E_{0}^{H F}}{\partial s}=0, \frac{\partial E_{0}^{H F}}{\partial \omega}=0$. As can easily be checked by a direct calculation, the above equations are equivalent to the so-called "gap equations":

$$
\begin{aligned}
& \frac{\partial E_{0}^{H F}}{\partial s}=0 \quad \Leftrightarrow \quad\langle\mathrm{HF}|[\mathrm{H}, b]| \mathrm{HF}\rangle=0, \\
& \frac{\partial E_{0}^{H F}}{\partial \omega}=0 \quad \Leftrightarrow \quad\langle\mathrm{HF}|[\mathrm{H}, b b]| \mathrm{HF}\rangle=0 .
\end{aligned}
$$

If $|\mathrm{HF}\rangle$ was the exact ground state, the above gap equations would be satisfied automatically. However, the deeper origin of these equations (minimization of the ground state energy with respect to the Bogoliubov parameters $\omega$ and $s$ ) implies that they have always to be satisfied.

Let us now discuss the RPA corrections to the ground state energy. In standard RPA the excitation operator $A$ entering the RPA equations, Eqs. (5), is taken to be $A \in\left\{b, b^{\dagger}, b b, b^{\dagger} b^{\dagger}\right\}$. We define the RPA ground state energy as follows:

$$
E_{0}^{\mathrm{RPA}}=\langle\mathrm{RPA}|\mathrm{H}| \mathrm{RPA}\rangle .
$$

This expression involves expectation values of the type $\langle\mathrm{RPA}|A B| \mathrm{RPA}\rangle$. These RPA corrected values can be calculated from the RPA Green's functions in the limit $t \rightarrow t^{\prime}$ :

$$
\langle\mathrm{RPA}|A B| \mathrm{RPA}\rangle=i \int \frac{d E}{2 \pi} G_{A B}(E) .
$$


It is in this way that we introduce RPA fluctuations in the calculation of the ground state energy.

In standard RPA the Green's functions on the right hand side of Eq. (16) are not determined self-consistently, i.e., the expectation values entering the Green's functions themselves are determined using the mean-field ground state $|\mathrm{HF}\rangle$. In our simple toy model we can obtain analytic expressions for the Green's functions.

Note that from Eqs. (国) we do not directly obtain Green's functions like $G_{x, x^{2}}$ or $G_{p, p}$, for example, since the operators $x, x^{2}$, and $p$ are not contained in our set of excitation operators $\left\{b, b^{\dagger}, b b, b^{\dagger} b^{\dagger}\right\}$. Hence, in order to apply Eq. (16) to expectation values like $\left\langle x^{3}\right\rangle$ or $\left\langle p^{2}\right\rangle$, the operators $x$ and $p$ are expressed in terms of $b$ and $b^{\dagger}$ (cf. Eq. (12)), and the resulting expectation values like $\langle b b b\rangle,\left\langle b^{\dagger} b b\right\rangle,\langle b b\rangle$, etc., are obtained from the Green's functions $G_{b, b b}, G_{b^{\dagger}, b b}, G_{b, b}$, etc.' 2 The short-hand notation $G_{x^{3}}$ or $G_{p^{2}}$ has to be understood as the corresponding linear combination of Green's functions like $G_{b, b b}$, $G_{b^{\dagger}, b b}, G_{b, b}$, etc., needed for the calculation of $\left\langle x^{3}\right\rangle$ or $\left\langle p^{2}\right\rangle$.

To determine $E_{0}^{\mathrm{RPA}}$ we need, in addition to $\left\langle x^{2}\right\rangle,\left\langle p^{2}\right\rangle$, and $\left\langle x^{3}\right\rangle$, also the expectation value $\left\langle x^{4}\right\rangle$. However, $G_{x^{4}}$ contains Green's functions of the type $G_{b^{\dagger}, b b b}$, that is, $A \in\left\{b, b^{\dagger}, b b, b^{\dagger} b^{\dagger}\right\}$ is not sufficient to calculate $\left\langle x^{4}\right\rangle$. We thus approximate this term by a factorization:

$$
\left\langle\mathrm{RPA}\left|x^{4}\right| \mathrm{RPA}\right\rangle \simeq 3\left\langle\mathrm{RPA}\left|x^{2}\right| \mathrm{RPA}\right\rangle^{2} .
$$

Finally, note that in the symmetric case $(s=0)$, the three-operator expectation values vanish which implies that the Green's function are the HartreeFock ones:

$$
i \int \frac{d E}{2 \pi} G_{x^{2}}^{s=0}(E)=\left\langle\mathrm{HF}\left|x^{2}\right| \mathrm{HF}\right\rangle
$$

Hence in this case the RPA energy is equal to the mean-field one.

In the literature the term RPA is used for a similar approximation to the Green's functions as described above. But, it has to be mentioned that often the charging formula 4, 15],

$$
E_{0}=E_{\mathrm{HF}}+\int_{0}^{1} \frac{d \lambda}{\lambda}\left\langle\lambda\left|\mathrm{H}_{\text {int }}(\lambda)\right| \lambda\right\rangle,
$$

\footnotetext{
${ }^{2}$ Note that there is an ambiguity in the assignment of the time arguments to some of the Green's functions. This does, however, not influence the results (see Appendix A for a more detailed discussion).

${ }^{3}$ Since the excitation operators have to be non-hermitian, two-particle operators of the form $b^{\dagger} b$ are not allowed. Thus we cannot use, e.g., $G_{b^{\dagger} b, b b}$ to determine $G_{x^{4}}$.
} 
is applied to determine the ground state energy. Thereby $\mathrm{H}_{\text {int }}(\lambda)=\lambda \mathrm{H}_{\text {int }}$ and the state $|\lambda\rangle$ is defined as the ground state with respect to $\mathrm{H}(\lambda)=$ $\mathrm{H}_{\text {bare }}+\lambda \mathrm{H}_{\text {int }}$ where $\mathrm{H}_{\text {int }}$ is the interacting part of the hamiltonian.

But, what we want to do is to compute directly $\langle g s|\mathrm{H}| g s\rangle$ with $|g s\rangle=$ $|\mathrm{HF}\rangle,|\mathrm{RPA}\rangle$ or $|0\rangle$ (see next section) and compare the result obtained within the different approximations. Thus again, $E_{0}^{\mathrm{RPA}}$ is not the energy as understood in standard RPA but directly $\langle\mathrm{RPA}|\mathrm{H}| \mathrm{RPA}\rangle$.

\section{The renormalized RPA approach}

What we want to do now is to go beyond the standard RPA approach and retain some of the correlations in the ground state. The starting point will be again the Bogoliubov transformation described in Sec. 3. In contrast to standard RPA we will, however, not use the mean-field ground state $|\mathrm{HF}\rangle$ but a more complicated one denoted $|0\rangle$. This new ground state, which will not be constructed explicitly in the following, contains some correlations. Since we want to use the "best" one, the parameters $\omega$ and $s$ will be again determined by the minimization of the ground state energy $E_{0}=\langle 0|\mathrm{H}| 0\rangle$. This leads to generalized gap equations:

$$
\begin{array}{ll}
\frac{\partial E_{0}}{\partial s}=0 \quad \Leftrightarrow \quad\langle 0|[\mathrm{H}, b]| 0\rangle=0, \\
\frac{\partial E_{0}}{\partial \omega}=0 \quad \Leftrightarrow \quad\langle 0|[\mathrm{H}, b b]| 0\rangle=0 .
\end{array}
$$

which are analogous to $\left\langle 0\left|\left[\mathrm{H}, Q_{\nu}^{\dagger}\right]\right| 0\right\rangle=0$ with the formalism introduced in Sec. 2. We may note that the equivalence between these formulas is no longer valid if we consider three particle excitation operators (see Sec. 5 for further details).

More precisely, independent of the approximation made to include correlations in the ground state, the above equations will guarantee that the basis is the energetically most favored one whithin this approximation.

In the remaining part of this article we will assume that all expectation values of the type $\langle 0|b b| 0\rangle,\left\langle 0\left|b b^{\dagger}\right| 0\right\rangle,\left\langle 0\left|b b b^{\dagger}\right| 0\right\rangle$, etc. are real. This implies for example that $\langle 0|p x| 0\rangle=-i / 2$ or $\left\langle 0\left|p x^{2}\right| 0\right\rangle=0$, independently of the specific ground state used. Thus, the key point is to determine the remaining expectation values in the a priori unknown ground state $|0\rangle$. In renormalized RPA [12, 16, 17, 18] this is achieved with the use of Green's functions in the 
limit $t \rightarrow t^{\prime}$. More precisely, we get self-consistency conditions for expectation values with two operators, i.e., $\langle 0|x x| 0\rangle$ and $\langle 0|p p| 0\rangle$ from

$$
\langle 0|A B| 0\rangle=i \int \frac{d E}{2 \pi} G_{A B}(E) .
$$

In contrast to Eq. (16), the expectation values entering the Green's functions on the right hand side of the above equation are determined self-consistently, i.e., not within the Hartree-Fock ground state. The remaining expectation values are approximated using a factorization, e.g.,

$$
\left\langle 0\left|p^{2} x^{2}\right| 0\right\rangle=\left\langle 0\left|p^{2}\right| 0\right\rangle\left\langle 0\left|x^{2}\right| 0\right\rangle+2\langle 0|p x| 0\rangle^{2} .
$$

This means that the self-consistent procedure is limited to expectation values with at most two operators and that the correlated part of the expectation values with more than two operators is neglected [4]. Moreover, since $\langle x\rangle$ vanishes, it follows immediately that terms with three operators such as, e.g., $\left\langle x^{3}\right\rangle$ vanish.

As for the standard RPA analytic expressions for the Green's functions can be given in terms of $\left\langle x^{2}\right\rangle,\left\langle p^{2}\right\rangle$ and $\langle p x\rangle$ (see Appendix A). The latter expectation values have then, in principle, to be determined numerically via the self-consistency conditions, Eq. (22). With these values at hand, we are able, in principle, to calculate the ground state energy $E_{0}$. However, renormalized RPA is known to miss an important part of the correlation energy [19]. Besides, this method cannot be derived via a variational principle and therefore the Ritz criterion is no longer applicable. In fact, what happens in most cases, is that the ground state obtains overbinding [19]. In our case it is even worse since one can show that a contradiction with the virial theorem and the Heisenberg uncertainty principle occurs. To that end let us look at the explicit equation for $\left\langle p^{2}\right\rangle$, which is obtained by integrating $G_{p^{2}}(E)$, given in Eq. (35), over E. The result is:

$$
\begin{array}{r}
\left\langle p^{2}\right\rangle=i \int \frac{d E}{2 \pi} G_{p^{2}}(E)=\epsilon^{2}\left\langle x^{2}\right\rangle \\
+i \frac{4(12 s g)^{2}\left\langle p^{2}\right\rangle}{\epsilon^{2}} \int \frac{d E}{2 \pi} \frac{1}{}\left(E^{2}-E_{1}^{2}\right)\left(E^{2}-E_{2}^{2}\right),
\end{array}
$$

where $\epsilon, E_{1}$ and $E_{2}$ are defined in Eq. (36). If we now look at the explicit form (Eq. (38)) of the gap equation for $\omega$ we conclude that the second term on the right hand side should vanish. However, this is only possible for $\left\langle p^{2}\right\rangle$ 
$=0$, implying a vanishing kinetic energy. This is obviously not a physically reasonable solution as it is in contradiction to the virial theorem. Even if we forget for the moment this contradiction, the self-consistent procedure can not give the right values for the occupation numbers and consequently for the energy. A possible way to circumvent that problem, that is to compute correctly the energy, is to use the "charging formula" as developed, e.g., in [4]. It is applied in a similar way as in standard RPA (cf. Sec. 3) to compute the correlation energy. The main difference is that we now have in principle a closed system of equations which have to be solved self-consistently. Following [4], one therefore replaces the gap equation concerning $s$, Eq. (20), by a minimization of the ground state energy obtained with the help of the charging formula (cf. Eq. (19)). This has of course one disadvantage: One loses the symmetry of the double commutators, i.e.

$$
\left\langle\left[b,\left[\mathrm{H}, b^{\dagger} b^{\dagger}\right]\right]\right\rangle-\left\langle\left[b^{\dagger} b^{\dagger},[\mathrm{H}, b]\right]\right\rangle=\left\langle\left[\mathrm{H}, b^{\dagger}\right]\right\rangle \neq 0 .
$$

This symmetry property, however, is crucial for the stability of the system [20]. We will therefore proceed in another direction.

\section{The extended r-RPA approach}

Instead of using the charging formula, our strategy to include further correlations in a minimal way will be as follows: We will abandon the factorization approximation for the lowest non-vanishing expectation values of products of more than two operators.

For the deformed case $(s \neq 0)$ this means that we will keep the values of $\left\langle x^{3}\right\rangle$ and $\left\langle p^{2} x\right\rangle$ (these are the only non-vanishing expectation values of three operators). Similar to $\left\langle x^{2}\right\rangle$ and $\left\langle p^{2}\right\rangle$, their actual values are determined via the self-consistency conditions, Eq. (22). With these expectation values at hand the ground state energy is then obtained, as before, as the expectation value of the Hamilton operator (cf. Eq. (13)):

$$
E_{0}^{s \neq 0}=\frac{\left\langle p^{2}\right\rangle}{2}+\frac{\mu^{2}}{2}\left(\left\langle x^{2}\right\rangle+s^{2}\right)+g\left(3\left\langle x^{2}\right\rangle^{2}+s^{4}+6 s^{2}\left\langle x^{2}\right\rangle-4 s\left\langle x^{3}\right\rangle\right) .
$$

First of all we have to note that there is no longer any obvious contradiction with the virial theorem. Moreover, as we will see in the next section, as far as we are not too close to the "phase transition", our results for the ground state 
energy are very close to the exact ones, without any further manipulation, like, e.g., the charging formula. This means $\left\langle x^{3}\right\rangle$ and $\left\langle p^{2} x\right\rangle$ contain already an important part of the so far missing correlations. This does not necessarily imply that we also reproduce the exact values for, e.g., $\left\langle x^{2}\right\rangle$ or $\left\langle p^{2}\right\rangle$. But the correlations are combined in such a way that the energy is determined very precisely within this approximation.

If we try to go further and include, e.g., also the correlated part of expectation values with four operators, we face serious problems. To obtain the Green's functions necessary to calculate $\left\langle x^{2}\right\rangle,\left\langle p^{2}\right\rangle,\langle p x\rangle,\left\langle x^{3}\right\rangle$ and $\left\langle p^{2} x\right\rangle$ it suffices to restrict the space of excitation operators to $A=\left\{b, b^{\dagger}, b b, b^{\dagger} b^{\dagger}\right\}$, i.e., two-particle excitations. As long as we stay in that space, the gap equations together with the assumption of real expectation values for $\left\langle b b^{\dagger}\right\rangle$, etc., guarantees the symmetry of the double commutators. As already discussed in Sec. 3, we need Green's functions of the type $G_{b^{\dagger}, b b b}$ to obtain, e.g., $\left\langle x^{4}\right\rangle$. That is, three particle excitation operators have to included. In addition to the (practical) computational problems we encounter another difficulty: As mentioned in Sec. 3, the Bogoliubov transformation contains only two parameters and consequently, including three particles implies a priori an approximation for the double commutators since there is no relation to enforce their symmetry. For instance, to ensure the following symmetry relation

$$
\left\langle\left[b b,\left[\mathrm{H}, b^{\dagger} b^{\dagger} b^{\dagger}\right]\right]\right\rangle-\left\langle\left[b^{\dagger} b^{\dagger} b^{\dagger},[\mathrm{H}, b b]\right]\right\rangle=6\left\langle\left[\mathrm{H}, b^{\dagger} b^{\dagger} b\right]\right\rangle,
$$

we needed an equation which guarantees $\left\langle\left[\mathrm{H}, b^{\dagger} b^{\dagger} b\right]\right\rangle=0$. As mentioned above the Bogoliubov transformation does not provide us with an additional adjustable parameter. The assumption of a generalized gap equation of the form $\langle[\mathrm{H}, Q]\rangle=0$ as used in many RPA approaches also did not help. In this context boson expansion techniques [3, 5], mapping a pair of bosons onto one new boson, could be helpful.

In the symmetric case $s=0$, it is known that, by parity, $\left\langle x^{3}\right\rangle$ and $\left\langle p^{2} x\right\rangle$ vanish. Following the same underlying philosophy as in the deformed case, we are now led to include $\left\langle x^{4}\right\rangle,\left\langle p^{4}\right\rangle$ and $\left\langle p^{2} x^{2}\right\rangle$. This is, as stated above, a subtle task since the double commutators need no longer to be symmetric. Fortunately, this difficulty can be overcome: Although in principle there are inherent problems with the symmetry of the double commutators, one can explicitly check that all non-symmetric terms disappear for $s=0$. This enables us to solve the Dyson equation without symmetrizing by hand (as it is often done in nuclear physics [20]). The corresponding Green's functions 
are listed in Appendix A. The expectation values $\left\langle x^{2}\right\rangle,\left\langle p^{2}\right\rangle,\left\langle x^{4}\right\rangle,\left\langle p^{4}\right\rangle$ and $\left\langle p^{2} x^{2}\right\rangle$ are then determined self-consistently using Eq. (22). In this case the ground state energy is given by:

$$
E_{0}^{s=0}=\frac{\left\langle p^{2}\right\rangle}{2}+\frac{\mu^{2}}{2}\left\langle x^{2}\right\rangle+g\left\langle x^{4}\right\rangle .
$$

As can be seen in the next section, the numerical results for the ground state energy are in very good agreement with the exact ones.

We have to emphasize that our approach is not fully self-consistent in the sense of SCRPA, although the expectation values of up to three or four operators, respectively, are calculated self-consistently. Let us mention one point which enlightens the difference of our method to the SCRPA approach used in Ref. [1]. The authors use the following form for the RPA excitation operator:

$$
Q^{\dagger}=\frac{1}{\sqrt{2}}\left(\lambda b^{\dagger} b^{\dagger}-\mu b b\right)
$$

This relation is easily inverted to give $b b$ and $b^{\dagger} b^{\dagger}$ in terms of $Q^{\dagger}$ and $Q$. Then the relation

$$
Q|S C R P A\rangle=0
$$

can be applied to show that, e.g., $\langle b b\rangle$ vanishes. Our excitation operator contains in addition one and three particle operators, proportional to $b, b^{\dagger}$ and $b b b, b^{\dagger} b^{\dagger} b^{\dagger}$, respectively. This renders the inversion less obvious and we therefore cannot directly make use of Eq. (29). Thus, a priori, we have to calculate several expectation values which can be shown to vanish within the SCRPA formalism of Ref. [11]. The SCRPA results for the ground state energy in the symmetric case are also in very good agreement with the exact ones [1].

\section{Numerical results}

This section is devoted to a discussion of the numerical results. In Fig. [ the energy of the ground state is displayed within the different approaches (exact result, mean-field, RPA, and our extended r-RPA result) as a function of $\mu^{2} / g$. Of course, even the mean-field is in reasonable agreement with the exact results (10\% at worst). The largest discrepancy can be observed in the region near the "phase transition" at $\mu^{2} / g \approx-6.8$, i.e., where $s$ jumps from 
some value $s \neq 0$ to $s=0$. This is also the case for the RPA and our r-RPA approach. But the figure clearly shows that the correlations incorporated in our approach are important: The agreement with the exact result is much better than in mean-field or RPA and discrepancies show up only in a very small region. Note that, the curve obtained within our approach is not continuous at the transition point. This is of course due to the difference in approximations made in the two domains: in one case, $s \neq 0$, one has incorporated the effect of $\left\langle x^{3}\right\rangle$ and $\left\langle p^{2} x\right\rangle$, whereas in the other case, $s=0$, we have included correlations in $\left\langle x^{4}\right\rangle,\left\langle p^{4}\right\rangle$ and $\left\langle p^{2} x^{2}\right\rangle$. Near the transition, also higher order correlations become important, and we therefore miss the exact result near the transition point.

Before we come to the conclusion, we would like to give one explicit example concerning the difference of our approach to the SCRPA: For $\mu^{2} / g=$ -1 , we obtain $\left\langle b^{\dagger} b b b\right\rangle=-0.033$ and $\left\langle b^{\dagger} b^{\dagger} b b\right\rangle=0.026$, i.e., both expectation values are of the same order of magnitude. In the SCRPA approach used in Ref. [11] the former expectation value vanishes identically.

\section{Conclusion}

We have considered an extended version of renormalized RPA that yields values of high accuracy for the ground state energy of the anharmonic oscillator. Moreover, the self-consistent procedure developed here removes contradictions present in renormalized RPA, that is $\left\langle x^{2}\right\rangle$ and $\left\langle p^{2}\right\rangle$ can be determined without evidently violating the virial theorem or the Heisenberg uncertainty principle.

This means that the correlations included, $\left\langle x^{3}\right\rangle$ and $\left\langle p^{2} x\right\rangle$ for $s \neq 0$ and $\left\langle x^{4}\right\rangle,\left\langle p^{4}\right\rangle$ and $\left\langle p^{2} x^{2}\right\rangle$ for $s=0$, represent the most important part of the correlations missed in renormalized RPA. Furthermore the systematics of the method allow us to think that it can be easily applied to quantum field theory and cure some of the problems which emerged in the work of Ref. 迎. One problem of our approach, on which we certainly should improve, is the fact that we do not have a unified approach for the two cases $s=0$ and $s \neq 0$.

Of course, it should be mentioned that there exist other powerful methods such as CCM (Coupled Cluster Method) [10] that describe very accurately the properties of the anharmonic oscillator. For problems concerning quantum chemistry or solid state physics, this technically and numerically very 


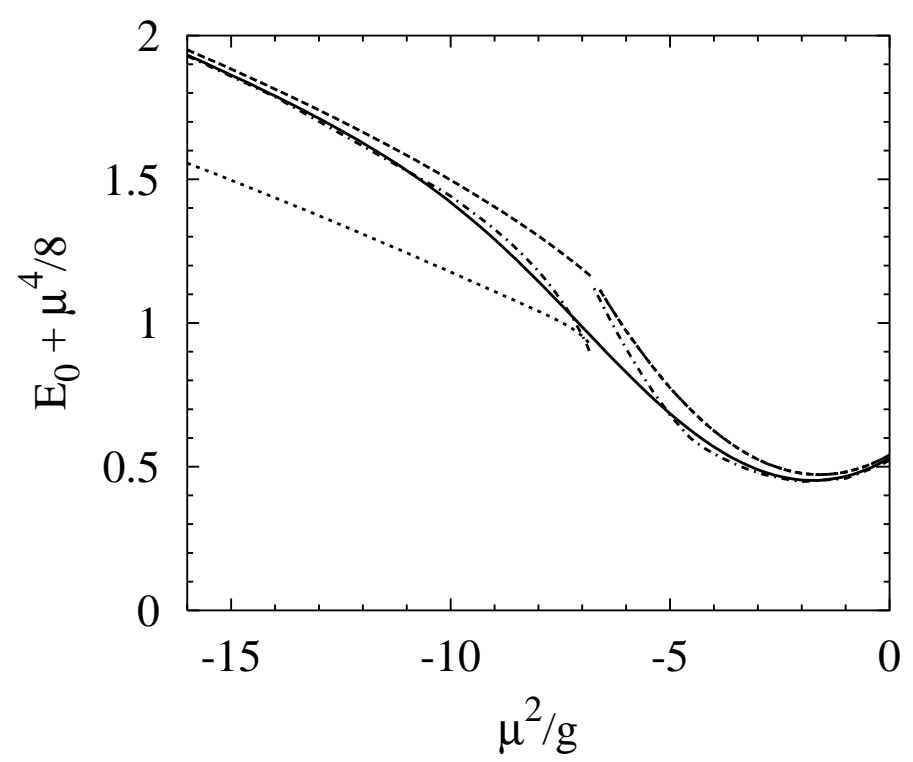

Figure 1: Exact solution for the ground state energy (solid line), mean field solution (dashed line), RPA solution (dotted line) and our extended r-RPA solution (dashed-dotted line) as a function of $\mu^{2} / g$ with $g=0.5$. Following [10], we added $\mu^{4} / 8$ in order to have positive energies. In the region where $s=0$ the mean-field and the RPA solution coincide (see text).

involved method and its extensions (see [10] for further details) are probably the most precise and well-suited. Actually, the approach developed in this paper probably can not compete with CCM in all these situations. Nevertheless it has the great advantage to identify clearly the underlying physics (correlations) and to be very easy to use.

\section{Acknowledgments:}

We gratefully acknowledge stimulating discussions with Z. Aouissat, and A. Rabhi. We also would like to thank G. Chanfray and P. Schuck for their constant interest in this work. One of us (M.O.) would like to thank the Alexander von Humboldt foundation for financial support. 


\section{A Explicit expressions for the Green's func- tions}

We present here the analytical results for the various Green's functions needed throughout the calculation. Note that the Green's function listed below do not directly emerge from the solution of the system of Eqs. (51) but we have to reexpress operators containing $x$ and $p$ with $b$ and $b^{\dagger}$, cf. Eqs. (12). For instance

$$
\begin{aligned}
G_{: x^{4}:}(E)= & \frac{1}{4 \omega^{2}} G_{:\left(b+b^{\dagger}\right)^{4}:}(E) \\
= & \frac{1}{4 \omega^{2}}\left(G_{b b, b b}(E)+G_{b^{\dagger} b^{\dagger}, b^{\dagger} b^{\dagger}}(E)+2 G_{b^{\dagger} b^{\dagger}, b b}(E)\right. \\
& \left.+4 G_{b^{\dagger}, b b b}(E)+4 G_{b^{\dagger} b^{\dagger} b^{\dagger}, b}(E)+4 G_{b^{\dagger} b^{\dagger}, b b}(E)\right) .
\end{aligned}
$$

One point is worth noting: We are dealing (in configuration space) with twotime Green's functions and the notation $G_{A, B}(E)$ indicates that the time arguments for the corresponding Fourier transformed quantity $G_{A, B}\left(t-t^{\prime}\right)$ are assigned as $A(t), B\left(t^{\prime}\right)$. This means that there is an ambiguity in the assignment of time arguments to the Green's functions which contain more than two operators. In most cases this can be overcome by the fact that our excitation operators are limited to non-hermitian ones, i.e. an operator like $b^{\dagger}(t) b(t)$ cannot exist. But, $G_{b b, b b}(E)$ as well as $G_{b, b b b}(E)$ do exist and in general they do not lead to the same expression. We are, however, only interested in the limit $t \rightarrow t^{\prime}$ to determine the expectation values at equal times. In this limit all functions coincide.

\section{A.1 Symmetric case}

In the symmetric case, i.e., $s=0$, we obtain

$$
\begin{aligned}
G_{x^{2}}(E) & =\frac{\left(E^{2}-\Sigma_{33}\right)-9 \frac{(\eta-\nu) \sigma^{2}}{4 \alpha_{3}^{2} \omega}}{\left(E^{2}-E_{1}^{2}\right)\left(E^{2}-E_{2}^{2}\right)}, \\
G_{p^{2}}(E) & =\frac{\epsilon^{2}\left(E^{2}-\Sigma_{33}\right)-9 \frac{(\eta+\nu)\left(\lambda-\omega^{3} \sigma\right)^{2}}{4 \alpha_{3}^{2} \omega^{5}}}{\left(E^{2}-E_{1}^{2}\right)\left(E^{2}-E_{2}^{2}\right)}, \\
G_{: x^{4}:}(E) & =\frac{1}{4 \omega^{2}}\left(-\frac{2\left(\lambda-2 g \omega+3 \omega^{3}\left(\alpha_{2} E+\Omega\right)\right)}{w^{3}\left(E^{2}-E_{01} E_{02}\right)}\right.
\end{aligned}
$$




$$
\begin{aligned}
& \left.+\frac{\tau}{4 \alpha_{3}^{2} \omega^{6}\left(E^{2}-E_{1}^{2}\right)\left(E^{2}-E_{2}^{2}\right)}\right), \\
G_{: p^{4}:}(E)= & \frac{\omega^{2}}{4}\left(-\frac{2\left(\lambda-2 g \omega+3 \omega^{3}\left(\alpha_{2} E+\Omega\right)\right)}{w^{3}\left(E^{2}-E_{01} E_{02}\right)}\right. \\
& \left.-\frac{\tau}{4 \alpha_{3}^{2} \omega^{6}\left(E^{2}-E_{1}^{2}\right)\left(E^{2}-E_{2}^{2}\right)}\right), \\
G_{: p^{2} x^{2}:(E)=} & \frac{\alpha_{2}}{2 \omega^{3}} \frac{E+E_{02}}{E^{2}-E_{01} E_{02}},
\end{aligned}
$$

where we have defined the following abbreviations:

$$
\begin{aligned}
& \epsilon^{2}=\mu^{2}+12 g\left\langle x^{2}\right\rangle \\
& \alpha_{2}=\frac{\left\langle p^{2}\right\rangle+\omega^{2}\left\langle x^{2}\right\rangle}{2 \omega} \text {, } \\
& \alpha_{3}=\frac{9}{2}\left\langle: p^{2} x^{2}:\right\rangle-3+\frac{9}{4 \omega^{2}}\left(\left\langle: p^{4}:\right\rangle+4\left\langle p^{2}\right\rangle \omega\right. \\
& \left.+4 \omega^{3}\left\langle x^{2}\right\rangle+\omega^{4}\left\langle: x^{4}:\right\rangle\right), \\
& \sigma=\left\langle p^{2}\right\rangle-\omega^{2}\left\langle x^{2}\right\rangle \text {, } \\
& \lambda=2 g\left(-3\left\langle p^{2}\right\rangle\left(1-2 \omega\left\langle x^{2}\right\rangle\right)+\omega(-2\right. \\
& -6\left\langle: p^{2} x^{2}:\right\rangle+3 \omega\left(5\left\langle x^{2}\right\rangle-2 \omega\left\langle x^{2}\right\rangle^{2}\right. \\
& \left.\left.\left.+2 \omega\left\langle: x^{4}:\right\rangle\right)\right)\right)+\omega\left(\omega^{2}-\epsilon^{2}\right) \sigma, \\
& \Sigma_{33}=\frac{\eta^{2}-\nu^{2}}{\alpha_{3}^{2}}, \\
& \eta=\left\langle\left[b b b,\left[\mathrm{H}, b^{\dagger} b^{\dagger} b^{\dagger}\right]\right]\right\rangle, \\
& \nu=\langle[b b b,[\mathrm{H}, b b b]]\rangle \text {, } \\
& \Omega=\frac{1}{\omega^{3}}\left(\lambda-4 \omega^{3}\left(\left\langle p^{2}\right\rangle+\epsilon^{2}\left\langle x^{2}\right\rangle\right)+22 g \omega\right. \\
& \left.+16 \omega^{2} g\left(-6\left\langle x^{2}\right\rangle+3 \omega\left\langle x^{2}\right\rangle^{2}-2 \omega\left\langle: x^{4}:\right\rangle\right)\right) \text {, } \\
& \tau=6 \alpha_{3}\left(9 \lambda^{2} \sigma+\lambda\left(-27 \sigma^{2}+4\left(\alpha_{3} E+\eta+\nu\right)(E-\omega)\right) \omega^{3}\right. \\
& +2 \sigma \omega^{5}\left(\omega \left(-4 E\left(\alpha_{3} E+\eta\right)+9 \sigma^{2}\right.\right. \\
& \left.\left.\left.+2\left(\alpha_{3} E+\eta+\nu\right) \omega\right)+2\left(\alpha_{3} E+\eta-\nu\right) \epsilon^{2}\right)\right), \\
& E_{01,2}=\frac{\Omega}{\alpha_{2}} \pm \frac{\lambda-2 g \omega}{\alpha_{2} \omega^{3}} \text {. }
\end{aligned}
$$


The energies $E_{1}^{2}$ and $E_{2}^{2}$ are the solution of the following equation:

$$
\begin{array}{r}
E^{4}+\frac{E^{2}}{4 \alpha_{3} \omega^{3}}\left(18 \sigma\left(\lambda-\sigma \omega^{3}\right)-4 \alpha_{3} \omega^{3}\left(\Sigma_{33}+\epsilon^{2}\right)\right) \\
+\frac{1}{16 \alpha_{3}^{2} \omega^{6}}\left(\left(81 \sigma^{2}+36 \omega(\eta+\nu)\right)\left(\lambda-\sigma \omega^{3}\right)^{2}\right. \\
\left.+\epsilon^{2} \omega^{5}\left(36 \sigma^{2}(\eta-\nu)+16 \alpha_{3}^{2} \omega \Sigma_{33}\right)\right)=0 .
\end{array}
$$

$\left\langle x^{3}\right\rangle$ and $\left\langle p^{2} x\right\rangle$ vanish because of parity. $\langle: \mathcal{O}:\rangle$ denotes the expectation value of the normal ordered product of the operator $\mathcal{O}$. If $|\mathrm{HF}\rangle$ is taken as ground state those expectation values obviously vanish and $\left\langle x^{2}\right\rangle=1 /(2 \omega),\left\langle p^{2}\right\rangle=$ $\omega / 2$. From the gap equations, Eqs. (20,21), we obtain the following condition

$$
\omega^{2}\left(\left\langle p^{2}\right\rangle-\epsilon^{2}\left\langle x^{2}\right\rangle\right)+3 g-12 g \omega\left\langle x^{2}\right\rangle\left(1-\omega\left\langle x^{2}\right\rangle\right)-4 g \omega^{2}\left\langle: x^{4}:\right\rangle=0 .
$$

\section{A.2 Deformed case}

In the deformed case, i.e. $s \neq 0$, we obtain:

$$
\begin{aligned}
G_{x^{2}}(E) & =\frac{E^{2}-\frac{\beta \kappa}{\alpha_{2}^{2}}}{\left(E^{2}-E_{1}^{2}\right)\left(E^{2}-E_{2}^{2}\right)} \\
& =\frac{1}{E^{2}-\epsilon^{2}+\frac{9 g^{2} \delta^{2}(\beta-\kappa)}{\omega^{2} \alpha_{2}^{2}} \frac{1}{E^{2}-\frac{\beta \kappa}{16 \omega^{4} \alpha_{2}^{2}}}}, \\
G_{p^{2}}(E) & =\epsilon^{2} G_{x^{2}}(E)+\frac{288 \delta^{2} g^{2} \kappa}{\alpha_{2}^{2}} \frac{1}{\left(E^{2}-E_{1}^{2}\right)\left(E^{2}-E_{2}^{2}\right)} \\
G_{: x^{3}:}(E) & =-\frac{6 \delta g\left(\alpha_{2}^{2} E^{2}+4 \omega \kappa+3 E\left(\kappa+\alpha_{2} \omega\right)\right)}{\alpha_{2} \omega^{2}\left(E^{2}-E_{1}^{2}\right)\left(E^{2}-E_{2}^{2}\right)} \\
G_{: p^{2} x:}(E) & =\frac{6 \delta g\left(\alpha_{2}^{2} E^{2}-E\left(\kappa+\alpha_{2} \omega\right)\right)}{\alpha_{2}\left(E^{2}-E_{1}^{2}\right)\left(E^{2}-E_{2}^{2}\right)},
\end{aligned}
$$

where we have introduced in addition the following abbreviations:

$$
\begin{aligned}
\epsilon^{2} & =\mu^{2}+12 g\left(s^{2}+\left\langle x^{2}\right\rangle\right) \\
\beta & =4\left(\left\langle p^{2}\right\rangle+\left\langle x^{2}\right\rangle\left(\epsilon^{2}+12 g\left\langle x^{2}\right\rangle\right)-18 g s\left\langle: x^{3}:\right\rangle\right) \\
\delta & =2 s\left\langle x^{2}\right\rangle-\left\langle: x^{3}:\right\rangle \\
\kappa & =2\left(\left\langle p^{2}\right\rangle\left(1+\frac{\epsilon^{2}}{\omega^{2}}\right)-12 g s\left\langle: x^{3}:\right\rangle+\left(\omega^{2}+\epsilon^{2}\right)\left\langle x^{2}\right\rangle\right.
\end{aligned}
$$




$$
\begin{aligned}
& \left.-\frac{24 g}{\omega^{2}}\left\langle: p^{2} x:\right\rangle s\right) \\
E_{1,2}^{2}= & \frac{1}{2}\left(\epsilon^{2}+\frac{\beta \kappa}{\alpha_{2}^{2}} \pm \sqrt{\left(\epsilon^{2}-\frac{\beta \kappa}{\alpha_{2}^{2}}\right)^{2}+8(12 g \delta)^{2} \frac{\kappa}{\alpha_{2}^{2}}}\right) .
\end{aligned}
$$

In this case we end up with two gap equations, one determining the value of $s$,

$$
s^{3}+s\left(3\left\langle x^{2}\right\rangle+\frac{\mu^{2}}{4 g}\right)-\left\langle: x^{3}:\right\rangle=0,
$$

and another one concerning $\omega$,

$$
\left\langle p^{2}\right\rangle=\epsilon^{2}\left\langle x^{2}\right\rangle-12 g s\left\langle: x^{3}:\right\rangle
$$

Note that the expression for $G_{x^{2}}(E)$ given in Eq. (35) can be interpreted as a free "propagator" with an additional "self-energy" depending on E. Similar interpretations are possible for all the other expressions.

\section{References}

[1] J.M. Häuser, W. Cassing, A. Peter, and M.H. Thoma, Z. Phys. A 353 (1995) 301.

[2] Z. Aouissat, G. Chanfray, P. Schuck, and J. Wambach, Nucl. Phys. A 603 (1996) 458.

[3] Z. Aouissat, P. Schuck, and J. Wambach, Nucl. Phys. A 618, 402 (1997).

[4] H. Hansen, G. Chanfray, D. Davesne, P. Schuck, Eur. Phys. J. A14 (2002) 397-411.

[5] Z. Aouissat, Phys. Rev. C 62 (2000) 012201.

[6] M. Oertel, M. Buballa, and J. Wambach, Phys. Atom. Nucl. 64 (2001) 698.

[7] G. Röpke, Z. Phys. B 99 (1995) 83;

J. Dukelsky and P. Schuck, Phys. Lett. B 387 (1996) 233.

[8] S. Schäfer, P. Schuck, Phys. Rev. B 59 (1999) 1712. 
[9] A. Rabhi, P. Schuck, R. Bennaceur, G. Chanfray, and J. Dukelsky, preprint nucl-th/0106064.

[10] R.F. Bishop and M.F. Flynn, Phys. Rev. A 38 (1988) 2211.

[11] J. Dukelsky and P. Schuck, Nucl. Phys. A 512 (1990) 466;

J. Dukelsky and P. Schuck, Mod. Phys. Lett. A 6 (1991) 2429.

[12] D.J. Rowe, Rev. Mod. Phys. 40 (1968) 153 and Nuclear Collective Motion, Methuen, London (1970).

[13] P. Schuck and S. Ethofer, Nucl. Phys. A212 (1973) 269.

[14] P. Schuck, J. Dukelsky, and G. Röpke, The Dyson equation approach to many body Green's functions, in Proceedings of the VI Hispalensis International Summer School, Oromana (Spain) June 1997, World Scientific, Singapore (1998).

[15] A.L. Fetter, J.D. Walecka Quantum Theory of Many-Particle Systems, McGraw-Hill, New York (1971).

[16] K. Hara, Progr. Theor. Phys. 32 (1964) 88.

[17] F. Catara, N. Dinh Dang and M. Sambatoro, Nucl. Phys. A 579 (1994) 1.

[18] J. Toivanen and J. Suhonen, Phys. Rev. Lett 75 (1995) 410.

[19] J. G. Hirsch, A. Mariano, J. Dukelsky, and P. Schuck Ann. Phys. 296 (2002) 187.

[20] M. Tohyama and P. Schuck, work in progress. 